\title{
Modelling expected physical impacts and human casualties from explosive volcanic eruptions
}

\author{
R. J. S. Spence ${ }^{1}$, I. Kelman ${ }^{1}$, E. Calogero ${ }^{1}$, G. Toyos $^{1}$, P. J. Baxter ${ }^{2}$, and J.-C. Komorowski ${ }^{3}$ \\ ${ }^{1}$ Department of Architecture, University of Cambridge, Cambridge, UK \\ ${ }^{2}$ Institute of Public Health, University of Cambridge, Cambridge, UK \\ ${ }^{3}$ Institut de Physique du Globe de Paris, CNRS UMR 7154, Volcanology Group, Paris, France
}

Received: 1 August 2005 - Revised: 26 September 2005 - Accepted: 10 October 2005 - Published: 19 December 2005

\begin{abstract}
A multi-hazard, multi-vulnerability impact model has been developed for application to European volcanoes that could significantly damage human settlements. This impact model is based on volcanological analyses of the potential hazards and hazard intensities coupled with engineering analyses of the vulnerability to these hazards of residential buildings in four European locations threatened by explosive volcanic eruptions. For a given case study site, inputs to the model are population data, building characteristics, volcano scenarios as a series of hazard intensities, and scenarios such as the time of eruption or the percentage of the population which has been evacuated. Outputs are the rates of fatalities, seriously injured casualties, and destroyed buildings for a given scenario. These results are displayed in a GIS, thereby presenting risk maps which are easy to use for presenting to public officials, the media, and the public. Technical limitations of the model are discussed and future planned developments are considered. This work contributes to the EU-funded project EXPLORIS (Explosive Eruption Risk and Decision Support for EU Populations Threatened by Volcanoes, EVR1-2001-00047).
\end{abstract}

\section{Introduction}

Several European settlements are at risk from the impact of explosive volcanic eruptions. A response to this threat is required from national and local Civil Protection authorities. Civil Protection agencies need to develop an understanding of both the scale of eruption which could take place (most probable scenarios and extreme but less probable scenarios) and their associated hazards. They need also to know what impact each possible style of eruption may have on the territory and the human settlements which it will affect, in terms of possible damage to buildings and other built facil-

Correspondence to: R. J. S. Spence

(robin@ carltd.com) ities along with casualties amongst the population who may remain in the area during an eruption.

Increasingly, eruption models are able to provide, for an assumed eruption scenario, a detailed map of the possible geographical distribution of the eruption products, with pointby-point estimates of the key parameters: depth and composition of tephra fall (Macedonio and Costa, 2005); dynamic pressure, velocity and particle concentration in pyroclastic flows (Esposti Ongaro et al., 2004); and time sequences of these variables. Where these parameters are known, it becomes possible to develop estimates of the impact of the eruption on buildings and infrastructure, and also on their occupants.

Previous studies, concentrating on Vesuvius, Italy have shown how, given an understanding of the distribution of a pyroclastic flow's dynamic pressure with distance from the volcano, the possible impact of an explosive eruption involving pyroclastic flows can be assessed, and how consequent human casualties might be estimated (Spence et al., 2004a, b). This work was based on an earlier 2-dimensional model of the volcano and the run-out of its eruption products (Esposti Ongario et al., 2002; Todesco et al., 2002).

Work under the EU-funded EXPLORIS project (Explosive Eruption Risk and Decision Support for EU Populations Threatened by Volcanoes, EVR1-2001-00047) has developed a new 3-dimensional eruption model for Vesuvius (Esposti Ongaro et al., 2004), as well as a new, more detailed tephra dispersion and fallout model for the same volcano (Macedonio and Costa, 2005). EXPLORIS also extends the eruption modelling to three other European volcanoes: Teide on the Spanish island of Tenerife, Soufrière on the French island of Guadeloupe, and Sete Cidades on São Miguel in the Portuguese Azores.

This volcanic hazard research has been complemented by the development of a new computer model for the estimation of the impacts on the potentially affected territories. This paper describes this model. During the project, it is anticipated to apply the model to look at impacts from possible eruptions at each of the four volcanoes, and to consider the 


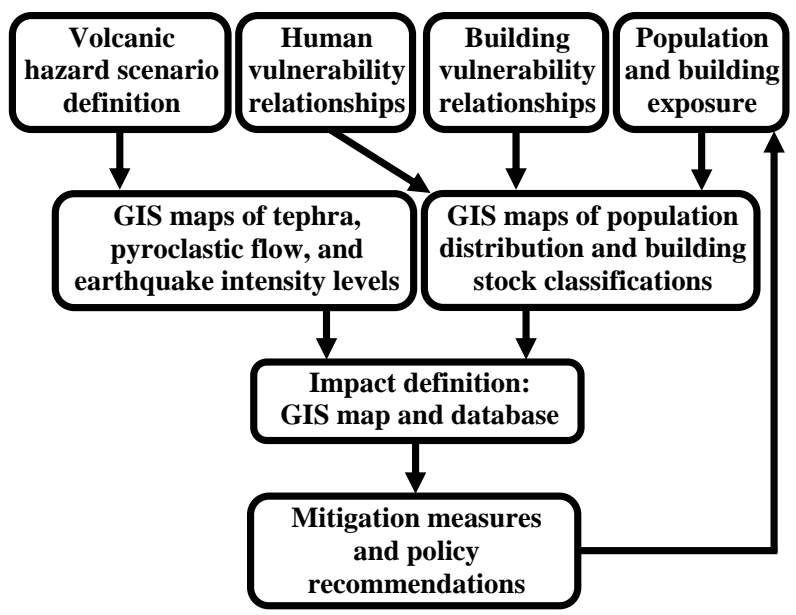

Fig. 1. The general structure of the impact model.

effectiveness of alternative mitigation options, producing results of direct relevance to the needs of Civil Protection agencies in each location.

The overall aims of the model are to develop a tool for estimating property damage and human casualties resulting from defined volcanic hazard scenarios. The model is designed to be applicable to the somewhat diverse conditions of the four volcanoes in terms of possible eruption styles and the types of buildings and settlements that could be affected. It is also designed to consider the impact of three separate types of volcanic hazards which can be expected to occur tephra fall, pyroclastic flows and volcanogenic earthquakes as well as to estimate property damage and human casualties. It is linked to a GIS mapping tool that enables maps of the inputs and the outputs to be displayed.

At the present time, the model is concerned with impacts on buildings (primarily residential buildings) and does not include effects on other infrastructure. It concentrates on hazards which are likely to accompany explosive eruptions, and therefore does not consider lava flows while also excluding post-eruption hazards such as floods, lahars and mudslides. Additionally, it produces a single composite estimate of damage and casualties as a result of an eruption which may last for some period of time.

In this paper, the model's structure is described and an example is given of applying the model to part of the potentially affected area for one of the four volcanoes, Soufrière of Guadeloupe. The structure of the data to input into the model is described; the way in which ground surveys were used to create the data needed is explained; the approach to vulnerability assessment for each of the hazards is briefly reviewed; and the results of two hypothetical eruption scenarios are presented. Other papers give more detail on the development of the vulnerability functions (Spence et al., 2005a, $\mathrm{b}^{1}$ ). Apply-

\footnotetext{
${ }^{1}$ Spence, R. J. S., Kelman, I., Brown, A., Purser, D., Baxter, P. J., and Calogero, E.: Residential building and occupant vulnerability to pyroclastic flow hazards, in preparation, 2005b.
}

ing the model to realistic eruption scenarios will be presented in future papers.

The work described in this paper was conducted within the EU-funded project EXPLORIS.

\section{Structure of the model}

Figure 1 shows the overall structure of the impact model. The impacted area is divided into several impact zones. The number of zones depends on the level of definition of the input data (on volcanic hazards, building stock, and population exposure) and on the output required. In the application described here, used to test the model, five impact zones in Guadeloupe were used. This is sufficient to capture the principal differences in building stock with limited hazard data available. As the volcanic hazard data become more detailed, a larger number of zones would be needed.

For each impact zone, three different types of input are required:

- Volcanic hazard scenario definitions,

- Exposure data for buildings and population,

- Vulnerability data for building damage and human casualties.

\subsection{Volcanic hazard scenario definitions}

The volcanic hazard scenario is defined in each zone by a single intensity value for each of the three principal hazards affecting building damage. A fourth intensity value primarily governs casualty generation, but does not significantly affect property damage.

The three hazards affecting building damage are tephra fall load, pyroclastic flow pressure, and earthquake ground shaking. An intensity scale for each of these three hazards has been devised, each intensity level defining a range of values of the principal measure of the parameter concerned. Ranges are not all of uniform interval, so that effects from the smallest hazard level, which will have some consequences, up to the largest foreseeable hazard level can be incorporated within a scale with a maximum of 7 scale points of 1 to 7 . Each intensity can also be set to zero, implying that this hazard is not present, or is insignificant, in the chosen scenario.

For tephra fall, the parameter chosen is vertical gravitational load acting on the roofs in the area, measured in Pa. It is the load which directly influences the roof damage. Tephra fall eruption models do not always define load; commonly, only tephra fall depth is calculated. In this case, an assumption needs to be made about the density of the fallen tephra, which may or may not be wet, or alternative assumptions made in order to calculate load. This value is given as an average value for the whole of the impact zone. The tephra fall intensity scale used is shown in Table 1. A tephra fall load of $10 \mathrm{kPa}$ (intensity 7) is likely to cause virtually all roofs to collapse (see Sect. 5.1). 
Table 1. Defined intensity levels for each hazard.

\begin{tabular}{lllllllll}
\hline Intensity level & 0 & 1 & 2 & 3 & 4 & 5 & 6 & 7 \\
\hline $\begin{array}{l}\text { Tephra fall load (kPa) } \\
\text { Pyroclastic flow pressure (kPa) }\end{array}$ & 0 & 1 & 2 & 3 & 4 & 6 & 8 & 10 \\
$\begin{array}{l}\text { External Heat Flux (EHF) } \\
\left(\min ^{\circ} \mathrm{C}\right)\end{array}$ & 0 & 200 & 4 & 1.5 & 2 & 3 & 4 & 6 \\
Earthquake Intensity (EMS) & 0 & $\mathrm{~V}$ & $\mathrm{VI}$ & $\mathrm{VII}$ & $\mathrm{VIII}$ & $\mathrm{IX}$ & & \\
\hline
\end{tabular}

For pyroclastic flow pressure, the pressure level which needs to be defined is the pressure at the level of ground floor windows (Pa), generally 1-2 $\mathrm{m}$ above ground floor level. This value, again, is given as an average value for the whole of the impact zone. The effects of urban sheltering and flow turbulence ensure that there is wide variation in this value, even within a short distance. The use of a single value is therefore controversial. Alternative models of the local effects of urban sheltering are currently being considered (Zuccaro and Calogero, $2005^{2}$ ), but a simple zonal model is not able to incorporate such detailed local variation at present. The model incorporates the assumption that the vertical profile of pyroclastic flow pressure, which is used to assess the impact on upper floor windows, is uniform. This assumption is discussed in Sect. 8. Table 1 shows the intensity scale used for pyroclastic flow pressure. The pressure associated with intensity 7 is not the maximum which could occur, but it would be enough to destroy most buildings in this area.

For earthquakes, the effects on buildings are reasonably well-defined by the use of well-known macroseismic intensity scales. This model uses the European Macroseismic Scale (EMS; Grünthal, 1998) which divides the level of ground shaking into 12 scale points, I to XII. Since the scale's lower levels do not affect buildings and since the upper levels are not thought likely to be experienced in volcanic eruptions, a truncated version of the scale has been adopted for this model which has then been renumbered to yield intensity levels (see Table 1). Again, a uniform intensity throughout the impact zone is assumed.

The fourth input parameter needed to define human casualties is the External Heat Flux (EHF) which is a measure of the combined effect of flow temperature and duration on the buildings affected. EHF governs the internal conditions and survivability for occupants and is fully defined in Sect. 6 . The EHF intensity scale adopted for this model is shown in Table 1. Again, an average value over the whole of the impact zone is required. An EHF value exceeding 2000 (intensity 7) would be lethal to all occupants, even in relatively wellsealed buildings.

\footnotetext{
${ }^{2}$ Zuccaro, G. and Calogero, E.: An Urban Sheltering Index for pyroclastic flow impacts estimation, in preparation, 2005.
}

\subsection{Exposure data}

For each impact zone, exposure data are required in terms of numbers of buildings and their occupants. Because the impact of each of the hazards on any building is dependent on the building's construction, several building classes have been defined, as described in Sect. 3. Typically, twenty separate classes of buildings need to be defined to capture all the important differences arising from different forms of construction, age and number of stories. Thus, the exposure data required are the number of buildings of each class in each zone. Occupant data are also needed, which is defined in terms of the number of occupants in each building.

The way in which exposure data are collected depends on the availability of existing building stock databases or mapping in any given location. This availability may influence the choice of impact zones. In any case, additional ground surveys are likely to be needed to identify all the important characteristics of each building class. The method implemented for Soufrière of Guadeloupe is discussed in Sect. 4.

\subsection{Vulnerability data}

To determine the impact of each volcanic hazard on the buildings and occupants, vulnerability relationships are required to estimate the effects at each intensity level for each hazard. These are discussed in Sects. 5 and 6. Each vulnerability relationship is specific to a building class and is applicable to the whole area, without zone-by-zone variation.

\subsection{Estimating impact}

Given the hazard intensity level, the buildings and occupants at risk, and the vulnerability relationships, estimating impacts for each building type and each zone is relatively straightforward. These can be summed to estimate the impact in each zone. A joint probability approach is used to sum impacts from the separate hazards, treating them as independent events. Implications are discussed in Sect. 7. The calculations are performed in an Excel spreadsheet.

Epistemic and aleatoric uncertainties in the model have been taken into account by allowing for each parameter (hazard, vulnerability and building classification) to be input as a probability distribution rather than as a single value, so that a range of impacts can be derived from multiple runs. GIS 
Table 2. Classes of construction material for the vertical loadbearing frame and their identifiers.

\begin{tabular}{lll}
\hline $\begin{array}{l}\text { Field } \\
\text { name }\end{array}$ & Type & $\begin{array}{l}\text { Other descriptive } \\
\text { notes }\end{array}$ \\
\hline CF & Reinforced concrete, infilled frame & \\
CS & Reinforced concrete, shear wall & \\
MB & Masonry, block/squared/cut stone & unreinforced \\
MC & Masonry, confined or reinforced & reinforced \\
MR & Masonry, rubble & \\
ST & Steel Frame & \\
TI & Timber & with lightweight \\
& & cladding \\
\hline
\end{tabular}

maps of the results can then be produced directly from the output tables. Examples are shown in Sect. 7.

\subsection{Evaluating mitigation options}

Mitigation actions which can be defined in terms of a change in one or more of the input parameters - building stock, occupancy or vulnerability - can be evaluated by examining the effects on the overall or local impacts of any given scenario. This can inform policy decisions such as the implementation of early warning systems and evacuation plans as well as long-term preventive and protective measures to buildings that can be managed by legislation such as building control or by insurance preconditions. Some possible options are discussed in Sect. 8.

\section{Classification of the building stock}

The aim of the building classification system was to devise a single system which could be used in all four of the EXPLORIS volcanoes and which would also be applicable to other locations. The approach needed to be sufficiently detailed to allow for the main variations between buildings affecting their response to the volcanic hazards, but not so detailed as to lead to a huge number of classes. From surveys of building stock for similar work in many locations (e.g. Pomonis et al., 1999), it has been found that three principal characteristics of a building are often primary, and that other characteristics can be assumed to be subsidiary, or can be derived from the primary classification. The three primary characteristics are:

- Materials of construction of the vertical load-bearing structure,

- Height (defined by number of storeys),

- Age.

For each primary characteristic, to keep total numbers of classes to an acceptably small number, a small number of alternatives needs to be defined.
Table 3. Classes of building height and their identifiers.

\begin{tabular}{cll}
\hline Field name & Number of storeys & Other descriptive notes \\
\hline $\mathrm{S}$ & 1 (single-storey) & Attics are considered as \\
$\mathrm{L}$ & 2 (low-rise) & an extension of the \\
$\mathrm{M}$ & $3,4,5$ (medium-rise) & storey below them, not \\
$\mathrm{H}$ & $6+$ (high-rise) & as an extra storey. \\
\hline
\end{tabular}

Seven classes of construction material were considered significant (Table 2). A two-letter code was adopted for each class. Each of these classes can be considered to have different responses to both pyroclastic flow pressure and to earthquakes.

Four alternative height ranges were distinguished (Table 3). The height of a building is significant for its response to pyroclastic flow pressure and for the impact of roof failure.

Four possible age-groups were identified to allow for change in the probable performance of each building type as a result of changes in building technology or regulations. The epochs of construction and the dates of significant change were different in different locations (Table 4).

By combining the field name codes for each primary characteristic, each class has a four-letter identifier. Thus, for the Vesuvius region, MRLO, a significant class in the area, refers to rubble masonry, low rise (two-storey), and built before 1920 . In theory, this classification system leads to a total of $7 \times 4 \times 4=112$ separate classes. In each area, some classes were empty or had insignificant numbers, leading to a total of around twenty significant separate classes for each volcano area.

Several characteristics of importance were not in the primary classification, such as the type and sizes of opening and roof construction. These characteristics were considered to be derived from the principal classes by means of a set of probability distributions, determined through field work and engineering calculations. Thus, the class MRLO was considered to have an 80/20 split of roofs between the class "weak" and the class "medium-weak", defining separate performance under tephra load (see Sect. 5).

Information on the distribution of primary and derived characteristics of the buildings in each volcanic area were derived by means of a field survey. An example of how such a survey was carried out, for a particular volcanic risk area, that of Soufrière Guadeloupe, is discussed in Sect. 4.

\section{Developing the GIS building stock inventory}

A field survey was undertaken for each of the four EXPLORIS volcanoes. This section presents a sample procedure carried out on Guadeloupe to examine possible impacts from an eruption of the Soufrière volcano. The results of the survey were then used to classify the building stock according to the three primary characteristics presented in Sect. 3 . 
Table 4. Age groups adopted for specific volcanoes and their identifiers.

\begin{tabular}{lcccc}
\hline Field name & Guadeloupe age band & Vesuvius age band & São Miguel age band & Tenerife age band \\
\hline O (old) & pre-1930 & pre-1920 & pre-1950 & pre-1930 \\
I (intermediate) & none & $1920-1940$ & none & none \\
M (modern) & $1930-1976$ & $1940-1990$ & $1950-1990$ & $1930-1980$ \\
R (recent) & post-1976 & post-1990 & post-1990 & post-1980 \\
\hline
\end{tabular}

Table 5. Data collected from building surveys.

\begin{tabular}{lll}
\hline Data collected & Sample categories & Reasons for collection \\
\hline Structural type & CF, CS, TI, MB, MR & See Sect. 3. \\
Building height & 4 categories & See Sect. 3. \\
Building age & recent, medium, old & See Sect. 3. \\
Air conditioning & present or not & For pyroclastic flow infiltration vulnerability. \\
Building use & residential or mixed & For population estimation. \\
Roof type & timber, concrete, coverings & For tephra strength (see Sect. 5). \\
Combustible materials & present or not & For fire hazard estimation. \\
Distance between buildings & $<6 \mathrm{~m}, 6-10 \mathrm{~m},>10 \mathrm{~m}$ & For fire hazard estimation. \\
Opening sizes & small $\left(<0.7 \mathrm{~m}^{2}\right)$ & For pyroclastic flow vulnerability. \\
& medium $\left(0.7-1.5 \mathrm{~m}^{2}\right)$ & \\
large $\left(>1.5 \mathrm{~m}^{2}\right)$ & For pyroclastic flow vulnerability. \\
Types of window frames & metal or timber & For pyroclastic flow vulnerability. \\
Type of shutters & good or poor & For pyroclastic flow vulnerability. \\
Condition of openings & &
\end{tabular}

The survey data were entered into a georeferenced database that could be analysed to give spatial and graphical representations of building class distributions by zone. This GISbased inventory would determine the exposure present in the areas most at risk to volcanic hazards. As a result, it would influence any impact estimation derived from it.

\subsection{Sample survey}

The survey was designed to collect data on not only the primary characteristics defined in Sect. 3, but also additional information about subsidiary characteristics of significance in assessing impacts. A list of the information collected is shown in Table 5. Such information was collected by sending out teams of two people to at least twenty predefined location points where twenty buildings for each location point were chosen to represent the area surveyed by the teams.

\subsection{Mapping procedure}

Survey location points were chosen and survey zones were defined by dividing the area under consideration, in this case Basse-Terre and St. Claude in Guadeloupe (see Fig. 2), into zones of comparable size that show differentiation in general urban morphological characteristics that also denote changes in construction typologies (see Fig. 2). In this case, it was considered that five zones would be sufficient for a robust characterisation of the distribution of building types present.

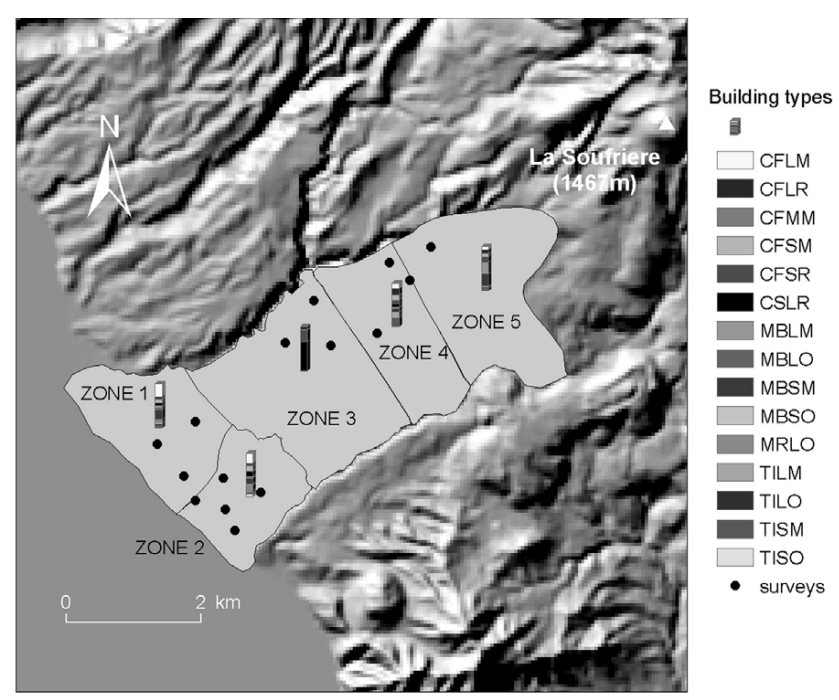

Fig. 2. Survey locations, vulnerability zones, and building class distribution in Basse-Terre and St. Claude on Guadeloupe.

\subsection{Example for Soufrière, Guadeloupe}

Figure 2 further shows the results of the field survey, illustrating the distribution of building classes among the five defined zones. The map shows that building typology distribution varies significantly from zone to zone, demonstrating the greater diversity of building classes in the downtown area 
Table 6. Classification of roofs, typical design loads and mean collapse loads estimated.

\begin{tabular}{llll}
\hline Roof class & Description & $\begin{array}{l}\text { Typical design } \\
\text { load range }\end{array}$ & $\begin{array}{l}\text { Mean collapse } \\
\text { load }\end{array}$ \\
\hline WE (weak) & $\begin{array}{l}\text { Sheet roofs, old or in poor condition. } \\
\text { Tiled roof, old or in poor condition. } \\
\text { Masonry vaulted roof. }\end{array}$ & $\begin{array}{l}\text { Pre-design code, } \\
\text { or no design } \\
\text { code. }\end{array}$ & $2.0 \mathrm{kPa}$ \\
MW (medium weak) & $\begin{array}{l}\text { Sheet roof on timber; average quality; average or good } \\
\text { quality tiled roof on timber rafters or trusses. } \\
\text { Steel or precast reinforced concrete joists and flat ter- } \\
\text { race roof. }\end{array}$ & $3.0 \mathrm{kPa}$ \\
MS (medium strong) & $\begin{array}{l}\text { Flat reinforced concrete roof not all above characteris- } \\
\text { tics; sloping reinforced concrete roof. }\end{array}$ & $2-3 \mathrm{kPa}$ & $4.5 \mathrm{kPa}$ \\
& $\begin{array}{l}\text { Sheet roof on timber rafters or trusses, good quality and } \\
\text { condition, designed for cyclone areas. } \\
\text { Flat reinforced concrete roof designed for access; re- } \\
\text { cent, good quality construction, younger than } 20 \text { years. }\end{array}$ & $>3 \mathrm{kPa}$ & $7.0 \mathrm{kPa}$ \\
\hline
\end{tabular}

of Basse-Terre (zones 1 and 2) with a relatively high proportion of old and weak building types and greater urban density. The suburban area between Basse-Terre and St. Claude with lower density shows a greater homogeneity of building types of more recent and robust construction (zone 3). St. Claude, a mix of both historic and recent settlements, also illustrates a diversity of building classes, but to a lesser degree than downtown Basse-Terre, with a higher proportion of robust and recent construction typologies (zones 4 and 5). Such information could play a significant role on the impact of a future eruption (see Sect. 7).

The complete residential building inventory in each impact zone was derived from a GIS map and building inventory made available by Guadeloupe's Department of Building and Planning (DDE Guadeloupe). From this map along with data from the INSEE (France) 1999 census for Guadeloupe, the number of residential buildings in each of the impact zones and the expected average building occupancy were determined. For each zone, the distribution of primary vulnerability classes was assumed to be the same as that of the buildings in the surveys carried out in that zone.

\section{Defining physical vulnerability of buildings}

Two different types of vulnerability needed to be defined, vulnerability of buildings to physical damage and vulnerability of occupants. Occupant vulnerability is in some respects dependent on physical damage to buildings and is considered in Sect. 6.

The physical vulnerability of buildings needs to defined in terms of damage states. A vulnerability function for a class of building defines the probability of that building being in a particular damage state, given the intensity level of the particular hazard concerned. The important hazards as far as physical damage is concerned (for explosive eruptions) are tephra fall, pyroclastic flow pressure, and earthquake ground shak- ing. A detailed account of the development of the vulnerability relationships for each of these hazards is given elsewhere, (Spence et al., 2005b $b^{1}$ ) so the account in this section will be summarised.

\subsection{Vulnerability to tephra fall}

The approach to tephra fall used is described in Spence et al. (2005a). Separate vulnerability relationships are defined for four classes of roofs, defined as weak, medium-weak, medium-strong and strong. The relationship of these general classes to the specific roof types found in the European area is shown in Table 6 . Table 6 also indicates the typical range of loads which the class might be designed for, and an estimate of the mean value of the collapse load.

The only damage state considered for tephra fall is the damage state of collapse, meaning "the failure of a major structural element" such that "the roof covering and the structural members supporting it will fall inwards along with the thick tephra layer above" (Spence et al., 2005a). The collapse damage state will be reached even if the roof collapse is confined to part of the building, e.g. one room. The focus is on this damage state because it is only roof collapse which leads to significant casualties. Figure 3 shows the vulnerability of each of the four roof classes as a function of tephra load (from Spence et al., 2005a).

\subsection{Vulnerability to pyroclastic flow}

Four physical damage states are of importance for assessing the impact of pyroclastic flow pressure. As pressure increases, the first damage state occurs when glazed openings start to fail, allowing pyroclastic flow materials to invade building interiors. A second damage state occurs when shuttered openings and solid doors fail. As pressure further increases, wall panels without opening may begin to fail, a third damage state. As pressure increases still further, roofs 
Table 7. Typical resistances of buildings and building elements to pyroclastic flow pressure (after Spence et al., 2004b).

\begin{tabular}{lc}
\hline Building elements & Expected resistance $(\mathrm{kPa})$ \\
\hline Window glass of ordinary buildings. & $1.0-2.5$ \\
Aluminium window in bad condition. & 1.5 \\
Aluminium window in good condition. & 3.0 \\
Old wooden door. & 3.5 \\
Old wooden window. & 5.0 \\
Weak non-aseismic reinforced concrete buildings (1 to 3 storeys). & $4.5-8$ \\
Terracotta tile in-fill panel without window. & 5.5 \\
Strong non-aseismic reinforced concrete buildings (4 to 7 storeys). & $5-9$ \\
Terra cotta tile in-fill panel with window. & $7.6-8.9$ \\
Weak aseismic reinforced concrete buildings (multi-storey). & $5-10$ \\
Tuff masonry wall (600 mm). & $10-13$ \\
Strong aseismic reinforced concrete buildings (multi-storey). & $6-14$ \\
Volcanic masonry wall (600 mm). & $20-26$ \\
\hline
\end{tabular}

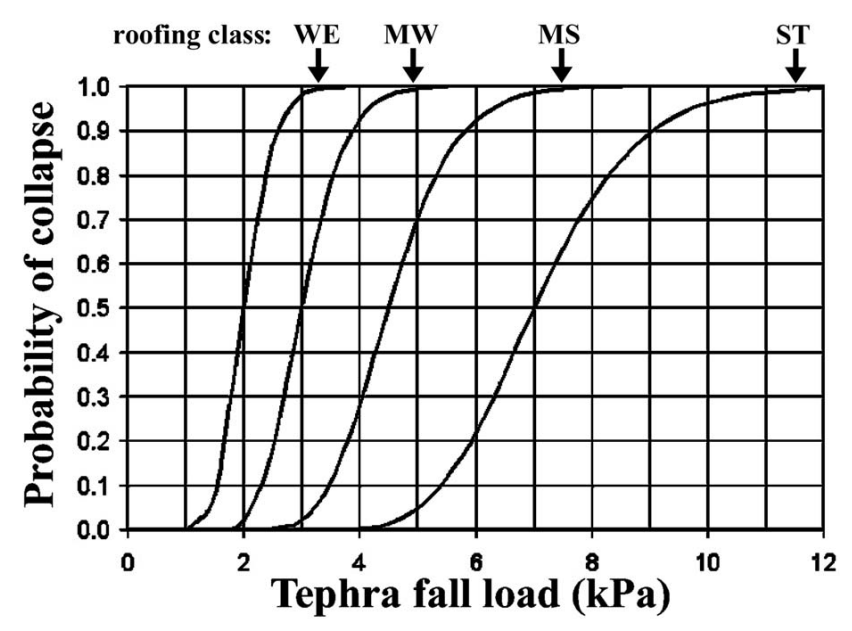

Fig. 3. Vulnerability curves for roof collapse from tephra fall (from Spence et al., 2005a).

and whole buildings may fail, the fourth damage state. Table 7 (after Spence et al., 2004b) shows in broad terms what level of damage to different classes of buildings or components can be expected as a result of increasing peak flow pressures.

The development of vulnerability relationships for each of these damage states, based on both calculation and on experiment, is given in Spence et al. (2004a, b). Further development is given in Spence et al. (2005b) ${ }^{1}$. Different vulnerability curves apply to different types of construction and sizes of opening.

Two important modifiers are temperature and missiles. The effect of a high flow temperature is to expand and distort glazed openings, reducing the lateral pressure needed to cause failure. Missiles carried by the pyroclastic flow will also increase the risk of glazing failure at any given flow pressure. The way in which these modifiers are taken into account is discussed elsewhere (Spence et al., 2005b ${ }^{1}$ ). Fig-

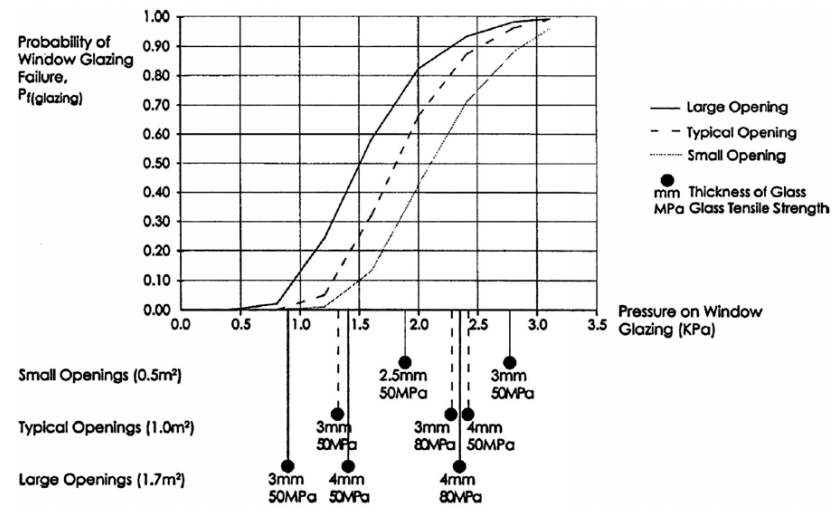

Fig. 4. Typical vulnerability curve for glazed openings (from Spence et al., 2004b).

ure 4 (from Spence et al., 2004b) shows an example of the vulnerability curves proposed for glazed openings. Figure 5 shows the curves for wall panel failure, based on particular conditions. Expected temperature and missile "availability" are needed as inputs to the model. Alternatively, a default can be used based on expert judgement.

\subsection{Vulnerability to earthquake}

For the purpose of estimating human casualties for earthquakes, the only damage states of importance are collapse and partial collapse. For the different classes of buildings found in Europe, damage probability matrices have been developed relating probability of collapse and partial collapse to the macroseismic intensity. These are presented, for the Vesuvian area, by Zuccaro et al. (2000). Table 8 shows characteristic data for rubble stone masonry buildings, giving probability of collapse or partial collapse, derived from the European Macroseismic Scale. 
Table 8. Probability of collapse or partial collapse of buildings of different vulnerability classes, based on the European Macroseismic Scale.

\begin{tabular}{ccccc}
\hline EMS vulnerability class & EXPLORIS building classes & Intensity VII & Intensity VIII & Intensity IX \\
\hline A & MR & $5 \%$ & $35 \%$ & $70 \%$ \\
B & MBO & $2 \%$ & $5 \%$ & $35 \%$ \\
C & MBR & $0 \%$ & $2 \%$ & $5 \%$ \\
D, E & CF, CS, TI & $0 \%$ & $0 \%$ & $2 \%$ \\
\hline
\end{tabular}

Table 9. Estimates of the human casualty consequences of volcanic hazards.

\begin{tabular}{|c|c|c|c|c|c|}
\hline Hazard & Building class & Condition & $\%$ killed & $\begin{array}{c}\% \text { seriously } \\
\text { injured }\end{array}$ & Comments \\
\hline \multirow[t]{2}{*}{ Tephra fall } & Single storey & Roof collapse & $16.7 \%$ & $16.7 \%$ & Of occupants of dwelling \\
\hline & Two or more stories & Roof collapse & $10 \%$ & $10 \%$ & Of occupants of top floor \\
\hline \multirow[t]{5}{*}{ Pyroclastic flow } & All types & One window fails & $15 \%$ & $15 \%$ & Of occupants of apartment \\
\hline & & Two windows fail & $40 \%$ & $40 \%$ & \\
\hline & & Three or more windows fail & $80 \%$ & $20 \%$ & \\
\hline & & Partial collapse of building & $50 \%$ & $50 \%$ & Of occupants of building \\
\hline & & Complete collapse of building & $100 \%$ & & \\
\hline \multirow[t]{3}{*}{ Earthquake } & Masonry & Collapse of building & $17.5 \%$ & $10 \%$ & Of occupants of building \\
\hline & Concrete frame & Collapse of building & $21 \%$ & $1 \%$ & \\
\hline & Timber & Collapse of building & $0.6 \%$ & $0.2 \%$ & \\
\hline
\end{tabular}

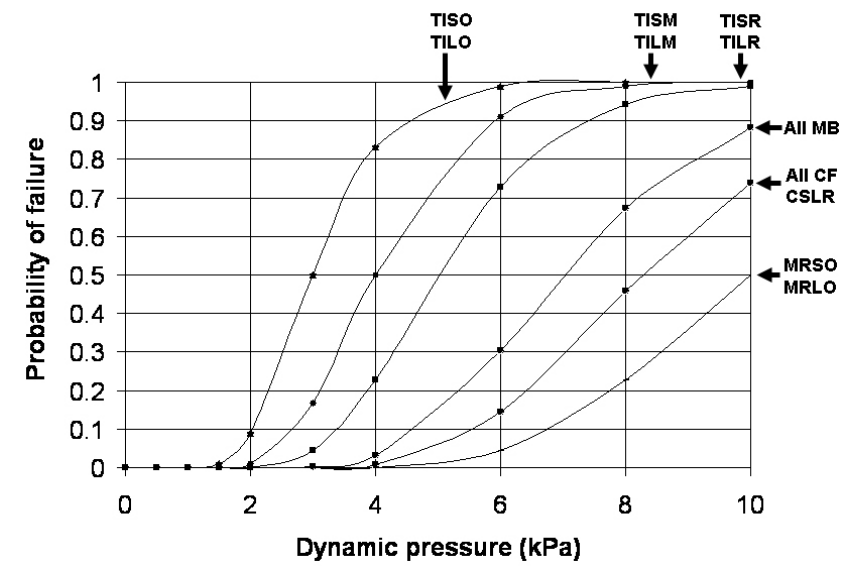

Fig. 5. Typical vulnerability curves for failure of masonry walls or infill wall panels under a pyroclastic flow's dynamic pressure.

\section{Human vulnerability}

Given that the main purpose of the model is to estimate potential loss of life and serious injury (injuries requiring medical intervention for survival) from the volcanic hazards, estimates are needed of the likely impacts on the building occupants at the time of the event or events. For the three hazards discussed in the previous section, estimates have been made of the consequences, in terms both of deaths and serious injuries, which would be likely to result from each level of physical damage. Table 9 summarises these estimates.

Justification for the casualty estimates presented in Table 9 is given elsewhere. For tephra fall casualties, estimates derive from studies of the effects of roof collapse in earthquakes, but also from evidence from the Mt Pinatubo eruption (Spence et al., 2005a). Estimates of casualties arising from invasion of ash into dwellings derive partly from observations of the effects of ash invasion in the Montserrat eruption (Baxter et al., 2005). Casualties arising from collapse or partial collapse in earthquakes derive from casualty studies in tectonic earthquakes (Coburn and Spence, 2002). Other background is given in Spence et al. (2004a, b, 2005b $\left.b^{1}\right)$.

One important source of expected casualties in a pyroclastic flow does not relate directly from building damage, but from the infiltration of the hot pyroclastic flow materials into the interior of buildings, through the normal ventilation process of air exchange between the inside and outside of a building. The flow's potential to cause death and injury has been found to relate both to the temperature and duration of the pyroclastic flow, and also to the building's ventilation rate.

A parameter, called the External Heat Flux (EHF), has been found to be a good predictor of the casualty rates for a building with a given ventilation rate. EHF is defined as $\mathrm{EHF}=\int_{t}(T-175) \mathrm{dt}$ where $T$ is the temperature in ${ }^{\circ} \mathrm{C}$ and $t$ is the time.

A preliminary estimate of the expected casualty rate from this infiltration hazard is given in Fig. 6, for buildings with three different levels of ventilation rate. The full justification of these curves and a discussion of the EHF concept is found in Spence et al. $(2005 b)^{1}$. 
Table 10. Case 1's model inputs, with the volcanic hazards defined as intensities (see Table 1).

\begin{tabular}{ccccccc}
\hline Zone & \# buildings & Population & Tephra fall & Pyroclastic flow pressure & Pyroclastic flow infiltration (EHF) & Earthquake \\
\hline 1 & 3231 & 8562 & 2 & 0 & 0 & 0 \\
2 & 2261 & 6191 & 2 & 0 & 0 & 0 \\
3 & 1245 & 2639 & 3 & 0 & 0 & 1 \\
4 & 674 & 1429 & 4 & 0 & 0 & 2 \\
5 & 795 & 1685 & 5 & 0 & 0 & 3 \\
Total & 8206 & 20507 & & & & \\
\hline
\end{tabular}

Table 11. Case 1's model outputs.

\begin{tabular}{ccccc}
\hline Zone & $\%$ buildings collapsed & \# buildings collapsed & $\%$ killed & \# killed \\
\hline 1 & $9 \%$ & 294 & $0 \%$ & 40 \\
2 & $12 \%$ & 263 & $1 \%$ & 51 \\
3 & $0 \%$ & 5 & $0 \%$ & 1 \\
4 & $45 \%$ & 302 & $5 \%$ & 77 \\
5 & $78 \%$ & 622 & $9 \%$ & 147 \\
Total & & 1487 & & 316 \\
\hline
\end{tabular}

\section{Sample results for hypothetical eruption scenarios}

Results for hypothetical eruption scenarios on Guadeloupe (see Fig. 2) are described in this section. The basis of such a prediction uses the building stock inventory described in Sect. 4, the vulnerability relationships described in Sects. 5 and 6, and an arbitrary designation of hazard for each zone intensity as set out in Sect. 3. These hazard inputs will need to be replaced with realistic hazard predictions when results are available. The hypothetical eruption scenarios used are not intended to be realistic. Further work will explore the application of the impact model to plausible eruption scenarios which are being developed within EXPLORIS based on the geological knowledge of the volcano's past activity (Komorowski et al., 2005) and additional detailed studies underway within EXPLORIS.

\subsection{Case 1}

In this hypothetical sample case, the hazard scenario corresponds to a volcanic eruption with volcanogenic earthquakes and tephra fall but with no pyroclastic flows. For tephra, the same intensity has been assumed in zones 1 and 2, while this increases in zones 3, 4 and 5, respectively. Earthquakes were assumed to affect zones 3, 4 and 5. The inputs have been set to values shown in Table 10. The outputs from the impact model are summarized in Table 11.

The results of the impact model for the first hypothetical scenario show that of 8206 buildings in the case study area, 1487 are predicted to collapse at a rate of $\approx 18 \%$ over the whole area surveyed. For the same area, the predicted number of fatalities is 316 from a population of 20507 , i.e. $\approx 2 \%$ of the population.

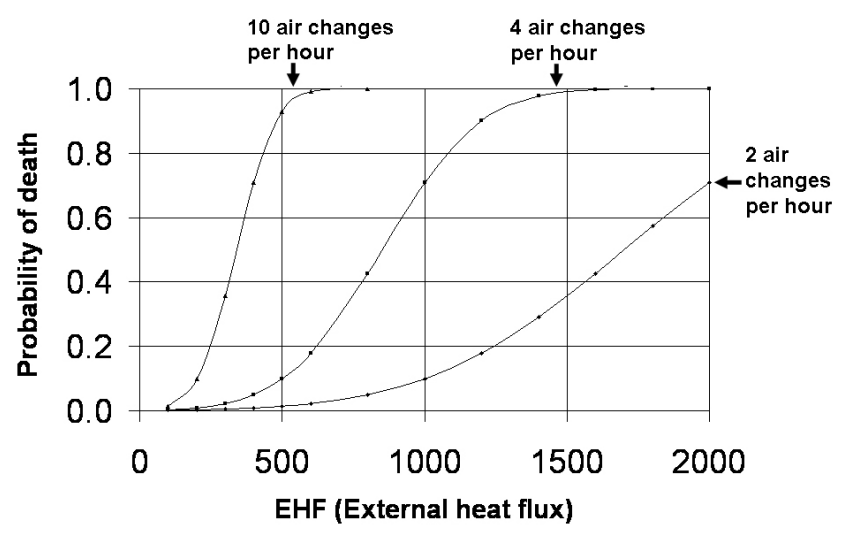

Fig. 6. Expected casualty rates from pyroclastic flow infiltration hazard for different ventilation rates.

The death and collapse rates vary from zone to zone. The minimum collapse rate is $0 \%$ and the maximum collapse rate is $78 \%$. The minimum death rate is $0 \%$ and the maximum death rate reaches $9 \%$. For this example, death and building collapse rates seem to follow a similar trend with a relatively stable proportional relationship between the two estimates. This is due to the way the model estimates deaths as a result of earthquake and tephra hazard, which is estimated on the basis of building collapse. Figure 7 shows the estimated building collapses and fatalities. Such a representation may be a useful way of presenting the results of impact estimates to emergency planners and strategic decision-makers. 
Table 12. Case 2's model inputs, with the volcanic hazards defined as intensities (see Table 1).

\begin{tabular}{|c|c|c|c|c|c|c|}
\hline Zone & \# buildings & Population & Tephra fall & Pyroclastic flow pressure & Pyroclastic flow infiltration (EHF) & Earthquake \\
\hline 1 & 3231 & 8562 & 2 & 4 & 4 & 0 \\
\hline 2 & 2261 & 6191 & 2 & 4 & 4 & 0 \\
\hline 3 & 1245 & 2639 & 3 & 5 & 5 & 1 \\
\hline 4 & 674 & 1429 & 4 & 6 & 6 & 2 \\
\hline 5 & 795 & 1685 & 5 & 7 & 7 & 3 \\
\hline Total & 8206 & 20507 & & & & \\
\hline
\end{tabular}

Table 13. Case 2's model outputs.

\begin{tabular}{ccccc}
\hline Zone & $\%$ buildings collapsed & \# buildings collapsed & $\%$ killed & \# killed \\
\hline 1 & $9 \%$ & 302 & $84 \%$ & 7165 \\
2 & $12 \%$ & 275 & $83 \%$ & 5142 \\
3 & $0 \%$ & 6 & $66 \%$ & 1746 \\
4 & $46 \%$ & 310 & $95 \%$ & 1352 \\
5 & $82 \%$ & 650 & $100 \%$ & 1679 \\
Total & & 1543 & & 17084 \\
\hline
\end{tabular}

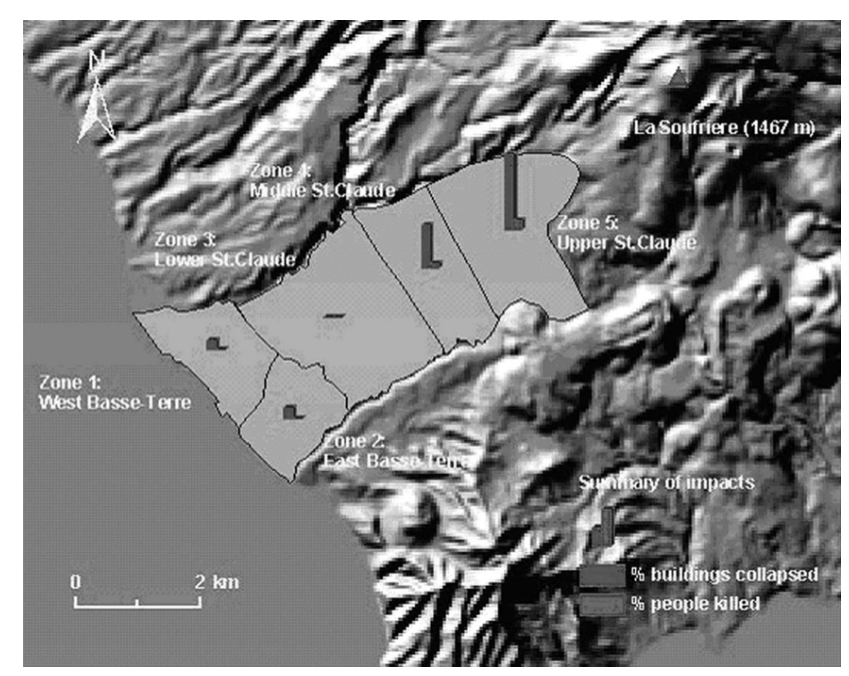

Fig. 7. Sample results for the Case 1 eruption scenario. For each zone, the bar on the left is the \% of buildings collapsed and the bar on the right is the $\%$ of people killed.

\subsection{Case 2}

In this hypothetical sample case, an eruption producing tephra fall, volcanogenic earthquakes and pyroclastic flows was assumed. The intensity of earthquakes and the tephra fall intensity have been left the same as in the previous case, so that the differences are solely due to the pyroclastic flow. The inputs have been set to values shown in Table 12 and the outputs from the impact model are summarized in Table 13.

The results of the impact model for the second hypothetical scenario show that of 8206 buildings in the case study area, 1543 are predicted to collapse at a rate of $19 \%$ over the whole area surveyed. This is only a small increase (by $1 \%$ ) over the results for Case 1 , showing that the number of building collapses caused by pyroclastic flows are much less than for those of the other two hazards combined. For the same area, the predicted number of fatalities in a population of 20507 is 17084 , i.e. $83 \%$ of the population. This is a huge leap from the estimates for a scenario without pyroclastic flows. The result highlights the devastation that would occur in an unevacuated area in the event of such a hazard, a result that is in agreement with the historical evidence (Blong, 1984).

The death and collapse rates vary from zone to zone. The minimum death rate is $66 \%$ and the maximum death rate is $100 \%$. These results do not seem to follow the same trend as the building collapse rate - which ranges between $0 \%$ and $82 \%$ - because death caused by pyroclastic flows are not dependent on building collapse. Figure 8 shows the estimated building collapses and fatalities.

Different eruption scenarios tested on this building stock vulnerability database show how some impacts are mitigated by the presence of certain types of building stock. For example, in both cases, zone 3 shows greater resilience to all three volcanic hazards. This seems to result from the dominance of concrete shear wall constructions in the area (see Sect. 4, Fig. 2). Estimated casualties are greater in areas such as zones 1 and 2 with lower hazard intensities due to the vulnerability of the building stock and the density of the population in those areas.

\section{Limitations of the model}

In its present stage of development, the model has several limitations which need to be factored in when considering the 
damage and human casualty estimates. These are discussed in this section.

First, it makes no allowance for damage and casualties from fires which might break out either within buildings or in the external environment as a result of the contact between hot pyroclastic flow materials and combustible building materials and contents. Such fires are expected when the temperature of the pyroclastic flow material is above about $300^{\circ} \mathrm{C}$. Their possible extent and consequences are a complex interaction between the temperature of flow materials, the combustibility and urban density of the building stock, the local vegetation and the environmental conditions. These issues are discussed by D. Purser in Kelman (2004) and will be considered in a simplified way in future model development.

Second, the model in its present stage takes no account of the effect of previous volcanic eruption impacts on the vulnerability of buildings to subsequent ones. For example, a damaging earthquake, even without complete destruction, will increase a building's vulnerability to a subsequent pyroclastic flow. Given a knowledge of the likely sequence of hazards, these effects of combined vulnerabilities can be taken into account, and work is currently being done to achieve this (G. Zuccaro et al.'s work in Kelman, 2004). However, in the present model, each of the three principal hazards is considered as an independent event, and the probability of damage or casualties from the combination of hazards has been calculated from a joint probability approach which ensures that deaths and injuries are not doublecounted.

Third, the vertical profile of the pyroclastic flow pressure on a building is considered uniform with height. Good reasons exist to believe that this assumption is inappropriate. Density of the flow materials is likely to increase towards ground level, but velocity will decrease towards ground level. Alternative shapes of pressure profile are possible depending on the relative importance of these two effects, but there is little direct evidence on which to base a better model. In a subsequent phase of the work, the impact of alternative plausible assumptions on the level of damage and casualties will be investigated.

A final limitation of the model is that the intensity of each of the hazards is averaged over a relatively large impact zone, within which variation will be significant for all hazards. A better model will not only look at substantially smaller impact zones than have been considered here, but will also consider the impact of urban sheltering on flow pressures and the effects of urban density on potential fire spread. On this topic too, further work is currently in progress which will be incorporated into future revisions of the impact model.

\section{Evaluating mitigation options}

As discussed above, the purpose of the impact model is to aid in evaluating and testing various mitigation options in order to determine the safest and most effective measures to

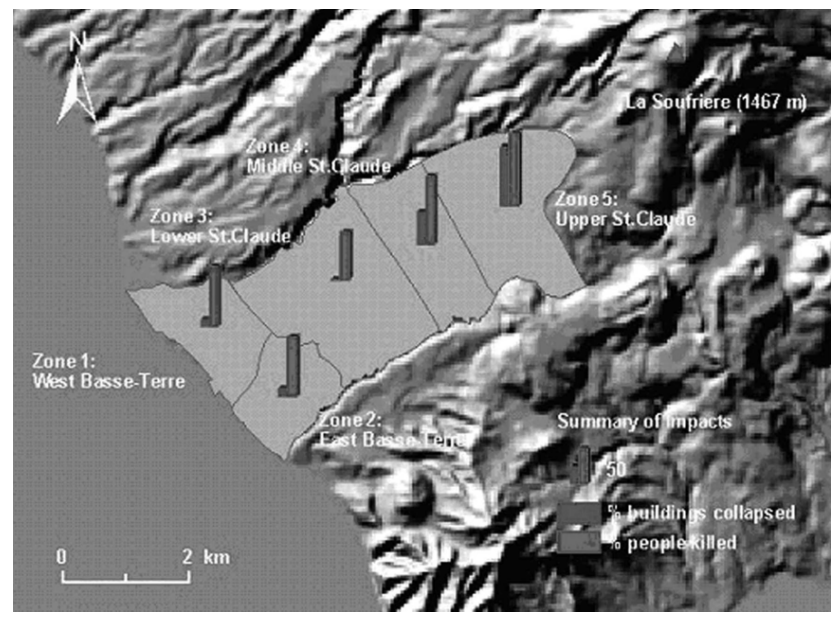

Fig. 8. Sample results for the Case 2 eruption scenario. For each zone, the bar on the left is the \% of buildings collapsed and the bar on the right is the $\%$ of people killed.

be taken to minimise the risk to human life in the event of an explosive volcanic eruption. This section discusses some possible measures, although further work on this subject is yet to be published (e.g. Spence et al., 2005b ${ }^{1}$ ). Here, three possibilities are investigated: evacuation, land-use planning and building control through legislation.

\subsection{Evacuation: short-term population displacement}

Short-term evacuation is the core of emergency plans for many volcanic areas. Where successful, it can produce a $100 \%$ survival rate with no casualties as a result of volcanic activity. This action was carried out in the last volcanic crisis on Guadeloupe in 1976 when 73422 people were officially evacuated from the danger zone, although it was estimated that as much as $3 \%$ of the population in the evacuated zone remained during the day and about $1 \%$ remained during the night (Lepointe, 1999).

Enforced evacuation of $100 \%$ of the population is feasible only if enough early warning is given where a sudden explosive volcanic eruption could leave the resident population exposed to a high risk of death. Potential false alarms, disbelief in warnings, inappropriate response actions, and legal action threats make the situation more complicated, meaning that future evacuation calls could be somewhat ineffective. The decisions of leaders in the time of crisis could be challenged, leading to the position of an unenforceable evacuation, an evacuation during an eruptive event, or an incomplete evacuation due to large numbers of people reluctant to evacuate.

Using the impact model could help in planning phased evacuations where time and resources are limited. It would allow the management of such an operation with the latest available hazard data in order to minimise the risk of death to the most vulnerable areas and to minimise casualty numbers. The impact model's quantitative probabilistic framework, closely linked to hazard and risk assessment, also 
offers a tool by which the benefits of mitigation decisionmaking could be readily assessed on more objective bases with confidence intervals. This is a major advantage which can help crisis managers to take key decisions in front of a potentially reluctant population or in the presence of conflicting interests.

\subsection{Land-use planning: long-term population displacement}

Long term land-use planning is another potential mitigation strategy that is already in use in the Vesuvius area: A new building moratorium is in place around the volcano and there are financial incentives for families to relocate outside of the zone deemed to be the most dangerous. Such a strategy takes a long-term view of reducing the risk to local populations around areas of volcanic hazard by reducing the exposure. Such strategies can be properly evaluated and measured by using the impact model which can evaluate the number of lives which would be saved as a result of long-term population relocation, in the event of a future eruption following the population relocation.

\subsection{Building control through legislation}

Another key risk reduction measure would be reinforcing and strengthening the building stock. In addition to preventing unnecessary economic loss, this option could save lives in the event of an eruption with little warning time. Such mitigation actions have been detailed elsewhere (Pomonis et al., 1999; Spence et al., 2004a, b, 2005a) and might include protecting openings, reducing ventilation rates, providing internal shelters, and providing short-term props for vulnerable roofs. The impact model would assist in analysing the effects on potential casualties if certain measures were widely adopted.

\section{Conclusions}

The impact model's results demonstrate how different hazard scenarios affect the potential impact of an eruption on the resident population within the defined hazard zones. The results from the model are preliminary and are considered as sample estimates of damage and casualties for hypothetical scenarios rather than as realistic, definitive predictions. This is due to the absence of realistic hazard data for model inputs as well as the fact that more research needs to be done into the relationship between casualties and fatalities for any given hazard. Any definitive results, would also include confidence intervals attached to all estimates that take into account aleatoric and epistemic uncertainties.

The method presented, however, shows the importance of having a procedural framework for systematically analysing the influence of a variety of hazard scenarios on the eventual impact of an eruption. It further illustrates how exposure varying across a geographic area, in terms of absolute population and resistance of that population to hazard, may influence decision-making in applying mitigating measures.
The format of the results, presented in GIS, should provide a valuable tool for civil protection and emergency planners to make decisions about short-term evacuation plans as well as longer-term disaster preparedness and planning.

Acknowledgements. The authors thank A. Neri, D. Purser, G. Zuccaro, IPGP, C. Badlou and staff from the Department of Buildings and Planning of the French Ministry of the Equipment (DDE) office in Basse-Terre for their ideas and information in support of this research. A. Brown, W. Fawcett, D. Haigh, C. Magill and J. Purser contributed to the field surveys. Topographic digital data, maps, and georeferenced airphotos were purchased from IGN (France). Population data is from the INSEE (France) 1999 census for Guadeloupe. A reviewer provided helpful suggestions and the EGU Editorial Office provided support. The European Union provided the funding which made this research possible through the project Explosive Eruption Risk and Decision Support for EU Populations Threatened by Volcanoes (EXPLORIS) EVR1-2001-00047.

Edited by: J. Marti

Reviewed by: R. Blong

\section{References}

Baxter, P. J., Boyle, R., Cole, P., Neri, A., Spence, R. J. S., and Zuccaro, G.: The impacts of pyroclastic surges on buildings at the eruption of the Soufrière Hills volcano, Montserrat, B. Volcanol., 67, 292-313, 2005.

Blong, R. J.: Volcanic Hazards: A Sourcebook on the Effects of Eruptions, Academic Press, London, UK, 1984.

Coburn, A. and Spence, R. J. S.: Earthquake Protection, John Wiley and Sons, 2002.

Esposti Ongario, T., Neri, A., Cavazzoni, C., and Erbacci, G.: 3D Multiphase Flow Simulation Outputs on a Large-Scale Domain, Deliverable D3.4 for the project Explosive Eruption Risk and Decision Support for EU Populations Threatened by Volcanoes (EXPLORIS) EVR1-2001-00047, INGV and CINECA, Italy, December, 2004.

Esposti Ongaro, T., Neri, A., Todesco, M., and Macedonio, G.: Pyroclastic Flow Hazard Assessment at Vesuvius by Using Numerical Modelling, 2. Analysis of flow variables, B. Volcanol., 64, 178-191, 2002.

Grünthal, G. (Ed.): European Macroseismic Scale, Council of Europe, Luxembourg, 1998.

Kelman, I. (Ed.): Residential Building and Occupant Vulnerability to Pyroclastic Flow, in Second Annual Report for the project Explosive Eruption Risk and Decision Support for EU Populations Threatened by Volcanoes (EXPLORIS) EVR1-200100047, Cambridge University Centre for Risk in the Built Environment, University of Cambridge, UK, December, 2004.

Komorowski, J.-C., Boudon, G., Semet, M., Beauducel, F., Anténor-Habazac, C., Bazin, S., and Hammouya, G.: Guadeloupe, in: Volcanic Atlas of the Lesser Antilles, edited by: Lindsay, J. M., Robertson, R. E. A., Shepherd, J. B., and Ali, S., Seismic Research Unit, The University of the West Indies, Trinidad and Tobago, 65-102, 2005.

Lepointe, E.: Le réveil du volcan de la Soufriére en 1976: la population guadeloupéenne à l'épreuve du danger, in: Les catastrophes naturelles aux Antilles - D'une Soufriére à l'autre, edited by: Yacou, A., CERC Université Antilles et de la Guyane, Editions Karthala, Paris, 15-71, 1999. 
Macedonio, G. and Costa, A.: 3D Simulation Outputs of the Mesoscale and Advection-Diffusion Models, Deliverable D3.10 for the project Explosive Eruption Risk and Decision Support for EU Populations Threatened by Volcanoes (EXPLORIS) EVR12001-00047, Osservatorio Vesuviano - INGV, Italy, January, 2005.

Pomonis, A., Spence, R., and Baxter, P. J.: Risk Assessment of Residential Buildings for an Eruption of Furnas Volcano, São Miguel, the Azores, J. Volcanol., 92, 107-131, 1999.

Spence, R. J. S., Baxter, P. J., and Zuccaro, G.: Building vulnerability and human casualty estimation for a pyroclastic flow: a model and its application to Vesuvius, J. Volcanol., 133, 321343, 2004a.

Spence, R. J. S., Zuccaro, G., Petrazzuoli, S., and Baxter, P. J.: Resistance of Buildings to Pyroclastic Flows: Analytical and Experimental Studies and Their Application to Vesuvius, Nat. Hazards Rev., 5, 48-59, 2004b.
Spence, R. J. S., Kelman, I., Baxter, P. J., Zuccaro, G., and Petrazzuoli, S.: Residential building and occupant vulnerability to Tephra Fall, Nat. Hazards Earth Syst. Sci., 5, 477-494, 2005b, SRef-ID: 1684-9981/nhess/2005-5-477.

Todesco, M., Neri, A., Esposti Ongaro, T., Papale, P., Macedonio, G., Santacroce, R., and Longo, A.: Pyroclastic Flow Hazard Assessment at Vesuvius by Using Numerical Modelling, 1. Large Scale Dynamics, J. Volcanol., 64, 155-177, 2002.

Zuccaro, G., Papa, F., and Baratta, A.: Aggiornamento delle mappe a scala nazionale di vulnerabilità sismica delle strutture edilizie, in: La vulnerabilità degli edifici: valutazione a scala nazionale della vulnerabilità sismica degli edifici ordinari, edited by: Bernardini, A., Gruppo Nazionale per la Difesa dai Terremoti, Rome, Istituto Nazionale di Geofisica e Vulcanologia, Italy, 2000. 